\title{
The violent collisional history of aqueously evolved (2) Pallas
}

Michaël Marsset ${ }^{1,2}$, Miroslav Brož ${ }^{3}$, Pierre Vernazza ${ }^{4}$, Alexis Drouard ${ }^{4}$, Julie Castillo-Rogez ${ }^{5}$, Josef Hanuš $^{3}$, Matti Viikinkoski ${ }^{6}$, Nicolas Rambaux ${ }^{7}$, Benoît Carry $^{8}$, Laurent Jorda ${ }^{4}$, Pavel Ševeček ${ }^{3}$, Mirel Birlan ${ }^{7}$, Franck Marchis ${ }^{9}$, Edyta Podlewska-Gaca ${ }^{10,11}$, Erik Asphaug ${ }^{12}$, Przemyslaw Bartczak ${ }^{10}$, Jérôme Berthier $^{7}$, Fabrice Cipriani ${ }^{13}$, François Colas ${ }^{7}$, Grzegorz Dudziński ${ }^{10}$, Christophe Dumas $^{14}$, Josef Ďurech $^{3}$, Marin Ferrais ${ }^{15}$, Romain Fétick ${ }^{4}$, Thierry Fusco ${ }^{4,16}$, Emmanuel Jehin ${ }^{15}$, Mikko Kaasalainen ${ }^{6}$, Agnieszka Kryszczynska ${ }^{10}$, Philippe Lamy ${ }^{4}$, Hervé Le Coroller $^{4}$, Anna Marciniak ${ }^{10}$, Tadeusz Michalowski ${ }^{10}$, Patrick Michel ${ }^{8}$, Derek C. Richardson ${ }^{17}$, Toni Santana-Ros ${ }^{18,19}$, Paolo Tanga $^{8}$, Frédéric Vachier ${ }^{7}$, Arthur Vigan ${ }^{4}$, Olivier Witasse ${ }^{13}$, Bin Yang $^{20}$

${ }^{1}$ Department of Earth, Atmospheric and Planetary Sciences, MIT, 77 Massachusetts Avenue, Cambridge, MA 02139, USA

${ }^{2}$ Astrophysics Research Centre, Queen's University Belfast, Belfast BT7 1NN, United Kingdom

${ }^{3}$ Institute of Astronomy, Charles University, Prague, V Holešovičkách 2, CZ-18000, Prague 8, Czech Republic

${ }^{4}$ Aix Marseille Univ, CNRS, CNES, Laboratoire d'Astrophysique de Marseille, Marseille, France

${ }^{5}$ Jet Propulsion Laboratory, California Institute of Technology, 4800 Oak Grove Drive, Pasadena, CA 91109, USA

${ }^{6}$ Mathematics and Statistics, Tampere University, 33720 Tampere, Finland

${ }^{7}$ IMCCE, Observatoire de Paris, 77 avenue Denfert-Rochereau, F-75014 Paris Cedex, France

${ }^{8}$ Université Côte d'Azur, Observatoire de la Côte d'Azur, CNRS, Laboratoire Lagrange, France 
${ }^{9}$ SETI Institute, Carl Sagan Center, 189 Bernado Avenue, Mountain View CA 94043, USA

${ }^{10}$ Astronomical Observatory Institute, Faculty of Physics, Adam Mickiewicz University, Słoneczna 36, 60-286 Poznań, Poland

${ }^{11}$ Institute of Physics, University of Szczecin, Wielkopolska 15, 70-453 Szczecin, Poland

${ }^{12}$ School of Earth and Space Exploration, Arizona State University, Tempe, AZ 85287, USA

${ }^{13}$ European Space Agency, ESTEC - Scientific Support Office, Keplerlaan 1, Noordwijk 2200 AG, The Netherlands

${ }^{14}$ TMT Observatory, 100 W. Walnut Street, Suite 300, Pasadena, CA 91124, USA

${ }^{15}$ Space sciences, Technologies and Astrophysics Research Institute, Université de Liège, Allée du 6 Août 17, 4000 Liège, Belgium

${ }^{16}$ ONERA, The French Aerospace Lab BP72, 29 avenue de la Division Leclerc, 92322 Chatillon Cedex, France

${ }^{17}$ Department of Astronomy, University of Maryland, College Park, MD 20742-2421, USA

${ }^{18}$ Departamento de Física, Ingeniería de Sistemas y Teoría de la Señal, Universidad de Alicante, E-03080 Alicante, Spain

${ }^{19}$ Institut de Ciències del Cosmos, Universitat de Barcelona (IEEC-UB), Martí i Franquès 1, E08028 Barcelona, Spain

${ }^{20}$ European Southern Observatory (ESO), Alonso de Cordova 3107, 1900 Casilla Vitacura, Santiago, Chile

Asteroid (2) Pallas is the largest main-belt object not yet visited by a spacecraft, making its surface geology largely unknown, and limiting our understanding of its origin and collisional 
evolution. Previous ground-based observational campaigns returned different estimates of its bulk density that are inconsistent with one another, one measurement ${ }^{1}$ being compatible within error bars with the icy Ceres $\left(2.16 \pm 0.01 \mathrm{~g} / \mathrm{cm}^{3}\right)^{2}$, and the other ${ }^{\frac{3}{3}}$ compatible within error bars with the rocky Vesta $\left(3.46 \pm 0.03 \mathrm{~g} / \mathrm{cm}^{3}\right)^{4}$. Here, we report high angular resolution observations of Pallas performed with the extreme Adaptive-Optics (AO)-fed SPHERE imager $^{\sqrt{5}}$ on the Very Large Telescope (VLT). Pallas records a violent collisional history, with numerous craters larger than $30 \mathrm{~km}$ in diameter populating its surface, and two large impact basins that could relate to a family forming impact. Monte-Carlo simulations of the collisional evolution of the main belt correlate this cratering record to the high average impact velocity of $\sim 11.5 \mathrm{~km} / \mathrm{s}$ on Pallas - compared with an average of $\sim 5.8 \mathrm{~km} / \mathrm{s}$ for the asteroid belt, induced by Pallas' high orbital inclination $\left(i=34.8^{\circ}\right)$ and orbital eccentricity $(e=0.23)$. Compositionally, Pallas' derived bulk density of $(2.89 \pm 0.08) \mathrm{g} / \mathrm{cm}^{3}$ is fully compatible with a CM chondrite-like body as suggested by its spectral reflectance in the 3-micron wavelength region $^{6}$. A bright spot observed on its surface may indicate an enrichment in salts during an early phase of aqueous alteration, compatible with Pallas relatively high albedo of 12-17\%, although alternative origins are conceivable.

We used the sharp angular resolution ( $\sim 20$ mas at $600 \mathbf{n m})$ of the SPHERE/ZIMPOL camera ${ }^{519}$ to characterize Pallas' bulk shape and surface properties with unprecedented details and, in turn, bringing new constraints on its origin and evolution. In total, 11 series of images were acquired during two apparitions as part of an ESO large program ${ }^{10}$. These images provide a full surface coverage, resolving $\sim 120$ to 130 pixels along Pallas' longest axis. The optimal angular resolu- 
tion of each image was restored with Mistral ${ }^{11 \mid 12}$, a myopic deconvolution algorithm optimized for images of objects with sharp boundaries, using a parametric point-spread function ${ }^{13}$.

The deconvolved images unveil a strong surface topographic relief suggestive of a violent collisional history (Fig.11). Numerous large ( $\sim 30-120-\mathrm{km}$ sized) impact features, including several craters with central peaks (Supplementary Fig. 1), are ubiquitous on Pallas, forming a surface reminiscent of a 'golf ball'. A total of 36 craters larger than $30 \mathrm{~km}$ in diameter $\left(D_{\mathrm{c}}\right)$ identified on the images (Fig. 2, Fig 3 and Supplementary Table 1), implies an observed average number density of $\mathrm{N}\left(D_{\mathrm{c}} \geq 40 \mathrm{~km}\right)=4.8 \pm 0.7 \times 10^{-5} \mathrm{~km}^{-2}$. The region with most favourable illumination in our observations (Fig. 3 ) is more than 3 times more cratered than this average, with $\mathrm{N}\left(D_{\mathrm{c}} \geq 40 \mathrm{~km}\right)=$ $1.6 \pm 0.2 \times 10^{-4} \mathrm{~km}^{-2}$, which seems comparable to the most heavily cratered geological units on Ceres ${ }^{14}$, and Vesta ${ }^{15}$ (see Methods). The similar maximum crater densities on Ceres, Pallas and Vesta could indicate some degree of saturation in this diameter range. It should be noted, however, that observed $D_{\mathrm{c}} \geq 40 \mathrm{~km}$ craters are relatively depleted on Ceres and Vesta, and the reported crater density values in this size range are often extrapolated from the observed number of smaller $\left(D_{\mathrm{c}} \geq 1 \mathrm{~km}\right)$ craters by use of a model production function ${ }^{16}$. In the case of Pallas, large craters are directly detected and cover a significant fraction (at least 9\%) of the total surface.

In order to understand the heavily cratered surface of Pallas, we explored its past collisional evolution, as well as that of the other two largest main-belt objects: Ceres and Vesta, through a series of Monte-Carlo simulations (see Methods). In each simulation, all collisional events capable of producing $D_{\mathrm{c}} \geq 40 \mathrm{~km}$ craters were recorded, using the $\pi$-scaling law ${ }^{17}$ to relate the crater 
diameter to the size of the impactor. The output of the simulations are shown in Fig. 4: The derived synthetic crater density on Pallas, $1.9 \pm 0.5 \times 10^{-4} \mathrm{~km}^{-2}$, turns out to be about 2 and 3 times larger than on Ceres and Vesta, respectively. Our simulations therefore hint towards the existence of even more cratered units on Pallas that are not seen in the SPHERE images. The results of our simulations directly reflect the different collisional environments and bulk properties of the three objects, including their size, bulk density, intrinsic collisional probability and, most importantly, average impact speed: while Ceres and Vesta are on rather circular and low-inclination orbits, Pallas' large orbital eccentricity $(e=0.23)$ and inclination $\left(i=34.8^{\circ}\right)$ imply typical impact velocities of $\sim 11.5 \mathrm{~km} / \mathrm{s}$ with other main-belt asteroids, versus $\sim 5.1$ and $5.3 \mathrm{~km} / \mathrm{s}$ for the other two bodies. Such large impact velocities of course drastically increase the number of projectiles able to create large craters owing to the steep size frequency distribution of the asteroid belt (slope approximately -2.5 in this size range $e^{\sqrt{18}}$ ). Specifically, the minimum impactor size needed to produce a $D_{\mathrm{c}} \geq 40 \mathrm{~km}$-size crater on Pallas is $\sim 2.4 \mathrm{~km}$, whereas it is comprised between $\sim 3.8$ and $\sim 4.3 \mathrm{~km}$ for the other two objects, implying a pool of 3 to 4 times more impactors for Pallas. This is only partially compensated by the lower intrinsic collisional probability between Pallas and impactors originating from the asteroid main belt. The heavily cratered surface of Pallas therefore appears to be a natural outcome of its peculiar orbit.

Next, the deconvolved images were fed to the ADAM algorithm $\frac{19}{19}$ together with previouslyacquired AO images from the Keck and VLT observatories (Supplementary Table 2), and optical light-curves (Supplementary Table 3), to precisely retrieve Pallas' spin orientation and 3D shape (see Methods). Direct comparison between the SPHERE images and projections of the resulting 
model are shown in Fig.2. The model has a volume-equivalent diameter of $D=513 \pm 6 \mathrm{~km}$. Semi-axes along the principal axes of inertia $(284 \times 266 \times 224) \pm 6 \mathrm{~km}$ indicate significant departure from hydrostatic equilibrium considering Pallas' current rotation period of $7.8 \mathrm{~h}$ (see Methods and Supplementary Fig. 2). This deviation can be explained by a substantial flattening of the South Pole of Pallas (Supplementary Fig. 3) that could relate to the existence of an ancient impact basin, similar to Rheasilvia on Vesta, and by a change of its rotation period, from $\sim 6.2 \mathrm{~h}$ to $7.8 \mathrm{~h}$, during such a basin-forming impact. The South-pole basin would represent $6 \pm 1 \%$ of the current volume of Pallas, which is significantly larger than the volume of Rheasilvia $(\sim 3 \pm 1 \%$ of the total volume of Vesta $\left.{ }^{20}\right)$. Another large excavation, roughly $1 \%$ the volume of Pallas, is found near its equator (Fig.2). Using a Smoothed-Particle Hydrodynamics (SPH) code to model the formation of the basins and their ejected fragments, we found that the size and volume of the equatorial basin are best reproduced assuming a large oblique impact with a 60-90-km-sized projectile (see Methods). Simulations of the subsequent orbital and collisional evolution of the resulting fragment population alines well both with the orbital distribution and size frequency distribution (SFD) of the current Pallas family after $1.7_{-0.4}^{+0.2}$ Ga evolution. This implies the equatorial basin could very well be the remnant of the Pallas family forming event. Similar simulations for the South-pole basin, on the other hand, suggest it does not relate to the present-day family.

Combining the volume measured from our 3-D shape model with available mass estimates (average value $(2.04 \pm 0.03) \times 10^{20} \mathrm{~kg}$; see Methods, Supplementary Fig. 4 and Supplementary Table 4) yields a density of $2.89 \pm 0.08 \mathrm{~g} / \mathrm{cm}^{3}$, significantly different from that of both Ceres $\left(2.16 \pm 0.01 \mathrm{~g} / \mathrm{cm}^{3}\right)^{2}$ and Vesta $\left(3.46 \pm 0.03 \mathrm{~g} / \mathrm{cm}^{3}\right)^{4}$, suggesting a distinct bulk composition for Pal- 
las. In particular, Pallas' higher density with respect to Ceres is most likely explained by a lower internal water-to-rock fraction, which is also consistent with Pallas' higher and seemingly more stable topography. Further, assuming an interior with little porosity, Pallas' density is fully compatible with the average grain density of $\mathrm{CM}$ chondrite meteorites $\left(2.90 \pm 0.08 \mathrm{~g} / \mathrm{cm}^{3}\right)^{21}$, Pallas' closest spectral analogues in the 3-micron spectral region ${ }^{6}$. This opens the possibility that Pallas accreted from the same starting material as the $\mathrm{CM}$-like $\mathrm{Ch}$ and Cgh-type asteroids ${ }^{22}$. In this scenario, spectral differences between these bodies over the visible and near-infrared wavelengths would result from distinct subsequent thermal and impact evolutions, owing to the larger size of Pallas and its unique collisional environment (see additional discussion in Methods).

A similar formation time for Pallas and the CM chondrites (3-4 Ma after the formation of Calcium-Aluminium-rich Inclusions, CAIs ${ }^{\sqrt{23}}$ would imply that the interior of Pallas never reached the silicate dehydration temperature $(\sim 820 \mathrm{~K})$ necessary to trigger the differentiation of a denser silicate core below a hydrated mantle, implying it has a rather homogeneous interior (see Methods and Supplementary Fig. 5). However, given Pallas' large size, partial differentiation (i.e., separation of water from silicates and upward flow) must have occurred in its interior, leading to an enrichment in salts that could explain Pallas' high albedo $\left(\mathrm{p}_{\mathrm{v}}=12-17 \%\right)^{7 / 8}$ with respect to Ch/Cgh-type asteroids $\left(\mathrm{p}_{\mathrm{v}}=6 \pm 2 \%\right)^{24}$. The presence of a bright spot with $\sim 10 \%$ brightness enhancement on Pallas (Fig. 11) reminiscent of those found on Ceres ${ }^{25}$, may provide additional support to the existence of salt deposits on its surface. However, alternative origins, such as the accretion of a bright exogenic material (e.g., ordinary chondrite) or the presence of unresolved ejecta blanket of a fresh impact that excavated bright material from the subsurface, cannot presently be ruled 
out. Considering that some studies proposed that the near-Earth object Phaethon originated

from Pallas ${ }^{26} 27$ (see discussion in Methods), we hypothesise that the presence of salts (therefore sodium) in Pallas is at the origin of the unusual diversity of sodium content measured in the Geminid meteors stream $28-32$ emitted by Phaethon ${ }^{33}$.

1. Schmidt, B. E. et al. The Shape and Surface Variation of 2 Pallas from the Hubble Space Telescope. Science 326, 275-278 (2009).

2. Park, R. S. et al. A partially differentiated interior for (1) Ceres deduced from its gravity field and shape. Nature 537, 515-517 (2016).

3. Carry, B. et al. Physical properties of (2) Pallas. Icarus 205, 460-472 (2010).

4. Russell, C. T. et al. Dawn at Vesta: Testing the Protoplanetary Paradigm. Science 336, 684$686(2012)$.

5. Beuzit, J. L. et al. SPHERE: the exoplanet imager for the Very Large Telescope. Astron. Astrophys. 631, A155 (2019). 1902.04080.

6. Larson, H. P., Feierberg, M. A. \& Lebofsky, L. A. The composition of asteroid 2 Pallas and its relation to primitive meteorites. Icarus 56, 398-408 (1983).

7. Tedesco, E. F., Noah, P. V., Noah, M. \& Price, S. D. The Supplemental IRAS Minor Planet Survey. Astron. J. 123, 1056-1085 (2002).

8. Alí-Lagoa, V., Müller, T. G., Usui, F. \& Hasegawa, S. The AKARI IRC asteroid flux catalogue: updated diameters and albedos. Astron. Astrophys. 612, A85 (2018). 1712.07496. 
9. Thalmann, C. et al. SPHERE ZIMPOL: overview and performance simulation. In Groundbased and Airborne Instrum. for Astron. II, vol. 7014 of Proc. SPIE, 70143F (2008).

10. Vernazza, P. et al. The impact crater at the origin of the Julia family detected with VLT/SPHERE? Astron. Astrophys. 618, A154 (2018).

11. Fusco, T. et al. Deconvolution of astronomical images obtained from ground-based telescopes with adaptive optics, vol. 4839 of Soc. of Photo-Optical Instrum. Eng. (SPIE) Conf. Series, 1065-1075 (2003).

12. Mugnier, L. M., Fusco, T. \& Conan, J.-M. MISTRAL: a myopic edge-preserving image restoration method, with application to astronomical adaptive-optics-corrected long-exposure images. J. Opt. Soc. Am. A 21, 1841-1854 (2004).

13. Fétick, R. J. et al. Closing the gap between Earth-based and interplanet. mission observations: Vesta seen by VLT/SPHERE. Astron. Astrophys. 623, A6 (2019). 1902 . 01287.

14. Scully, J. E. C. et al. Ceres' Ezinu quadrangle: a heavily cratered region with evidence for localized subsurface water ice and the context of Occator crater. Icarus 316, 46-62 (2018).

15. Schmedemann, N. et al. The cratering record, chronology and surface ages of (4) Vesta in comparison to smaller asteroids and the ages of HED meteorites. Planet. Space Sci. 103, 104-130 (2014).

16. Hiesinger, H. et al. Cratering on Ceres: Implications for its crust and evolution. Science 353, aaf4759-1-aaf4759-8 (2016). 
17. Melosh, H. J. Impact cratering: A geologic process (New York, Oxford University Press, 1989).

18. Gladman, B. J. et al. On the asteroid belt's orbital and size distribution. Icarus 202, 104-118 (2009).

19. Viikinkoski, M., Kaasalainen, M. \& Durech, J. ADAM: a general method for using various data types in asteroid reconstruction. Astron. Astrophys. 576, A8 (2015).

20. Schenk, P. et al. The Geologically Recent Giant Impact Basins at Vesta's South Pole. Science 336, 694-697 (2012).

21. Consolmagno, G., Britt, D. \& Macke, R. The significance of meteorite density and porosity. Chem Erde-Geochem 68, 1-29 (2008).

22. Vernazza, P. et al. Compositional Homogeneity of CM Parent Bodies. Astron. J. 152, 54 (2016).

23. Doyle, P. M. et al. Early aqueous activity on the ordinary and carbonaceous chondrite parent bodies recorded by fayalite. Nat. Commun. 6, 7444 (2015).

24. Masiero, J. R. et al. Preliminary Analysis of WISE/NEOWISE 3-Band Cryogenic and Postcryogenic Observations of Main Belt Asteroids. Astrophys. J. 759, L8 (2012). 1209. 5794.

25. Nathues, A. et al. Sublimation in bright spots on (1) Ceres. Nature 528, 237-240 (2015).

26. de León, J., Campins, H., Tsiganis, K., Morbidelli, A. \& Licandro, J. Origin of the near-Earth asteroid Phaethon and the Geminids meteor shower. Astron. Astrophys. 513, A26 (2010). 
27. Todorović, N. The dynamical connection between Phaethon and Pallas. Mon. Not. R. Astron. Soc. 475, 601-604 (2018). 1801.08857 .

28. Trigo-Rodríguez, J. M., Llorca, J., Borovička, J. \& Fabregat, J. Spectroscopy of a Geminid Fireball: its Similarity to Cometary Meteoroids and the Nature of its Parent Body. Earth Moon Planets 95, 375-387 (2004).

29. Borovička, J., Koten, P., Spurný, P., Boček, J. \& Štork, R. A survey of meteor spectra and orbits: evidence for three populations of Na-free meteoroids. Icarus 174, 15-30 (2005).

30. Borovička, J. Spectroscopic Analysis of Geminid Meteors. In Rendtel, J. \& Vaubaillon, J. (eds.) Proc. of the Int. Meteor Conf., 26th IMC, Bareges, France, 2007, 42-51 (2010).

31. Kasuga, T., Watanabe, J. \& Ebizuka, N. A 2004 Geminid meteor spectrum in the visibleultraviolet region. Extreme Na depletion? Astron. Astrophys. 438, L17-L20 (2005).

32. Kasuga, T. Thermal Evolution of the Phaethon-Geminid Stream Complex. Earth Moon Planets 105, 321-326 (2009).

33. Whipple, F. L. 1983 TB and the Geminid Meteors. IAU Cirulars 3881 (1983).

Acknowledgements Based on observations collected at the European Organisation for Astronomical Research in the Southern Hemisphere under ESO programme 199.C-0074 (PI: P.V.). This research has made use of the Keck Observatory Archive (KOA), which is operated by the W. M. Keck Observatory and the NASA Exoplanet Science Institute (NExScI), under contract with the National Aeronautics and Space Administration. M.M. was supported by the National Aeronautics and Space Administration under Grant 
No. 80NSSC18K0849 issued through the Planetary Astronomy Program. This work was supported by the French Direction Générale de l'Armement (DGA) and Aix-Marseille Université (AMU). P.V., A.D. and B.C. were supported by CNRS/INSU/PNP. J.H., J.Ď. and P.Š. were supported by the grant 18-09470S of the Czech Science Foundation and by the Charles University Research Programme No. UNCE/SCI/023. M.Brož was supported by the grant 18-04514J of the Czech Science Foundation. This project has received funding from the European Union's Horizon 2020 research and innovation programme under grant agreement No. 730890. This material reflects only the authors' views and the Commission is not liable for any use that may be made of the information contained herein.

Author contributions P.V. is the Principal Investigator of the ESO large survey that acquired the images of Pallas. M.M. and P.V. designed and operated the survey in service mode. M.M. led the research on Pallas. M.M., P.V., R.F. and T.F. reduced and deconvolved the SPHERE images. A.D. performed the crater analysis. M.Brož analysed the Pallas family and ran the N-body and SPH simulations. D.C.R and E.A. provided some of the numerical codes used for the simulations. B.C. and J.H. retrieved earlier disk-resolved and disk-integrated data for Pallas from the litterature. M.V. and J.H. reconstructed the 3D shape of Pallas. N.R. and L.J. analysed the shape. B.C. provided the mass estimate. J.C. performed the compositional analysis and thermophysical modelling of Pallas. M.M., M.Brož, P.V. and J.C. worked jointly to write the manuscript. All authors discussed the results and commented on the manuscript.

Competing Interests The authors declare that they have no competing financial interests.

Correspondence Correspondence and requests for materials should be addressed to M. Marsset (email: mmarsset@mit.edu). 


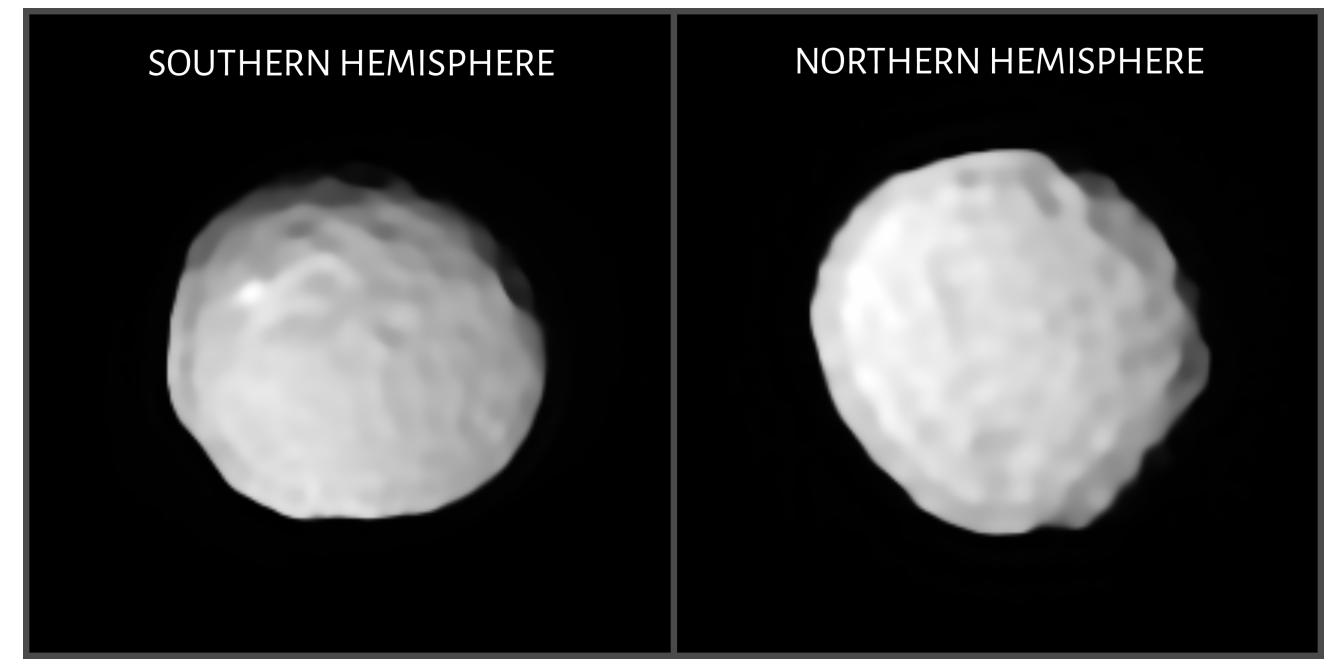

Figure 1: The two hemispheres of (2) Pallas as seen by VLT/SPHERE. Images taken on UT October 282017 (southern hemisphere) and UT March 152019 (northern hemisphere). Numerous large craters are visible on both hemispheres, and a bright spot reminiscent of salt deposits on Ceres is found on the southern one. 


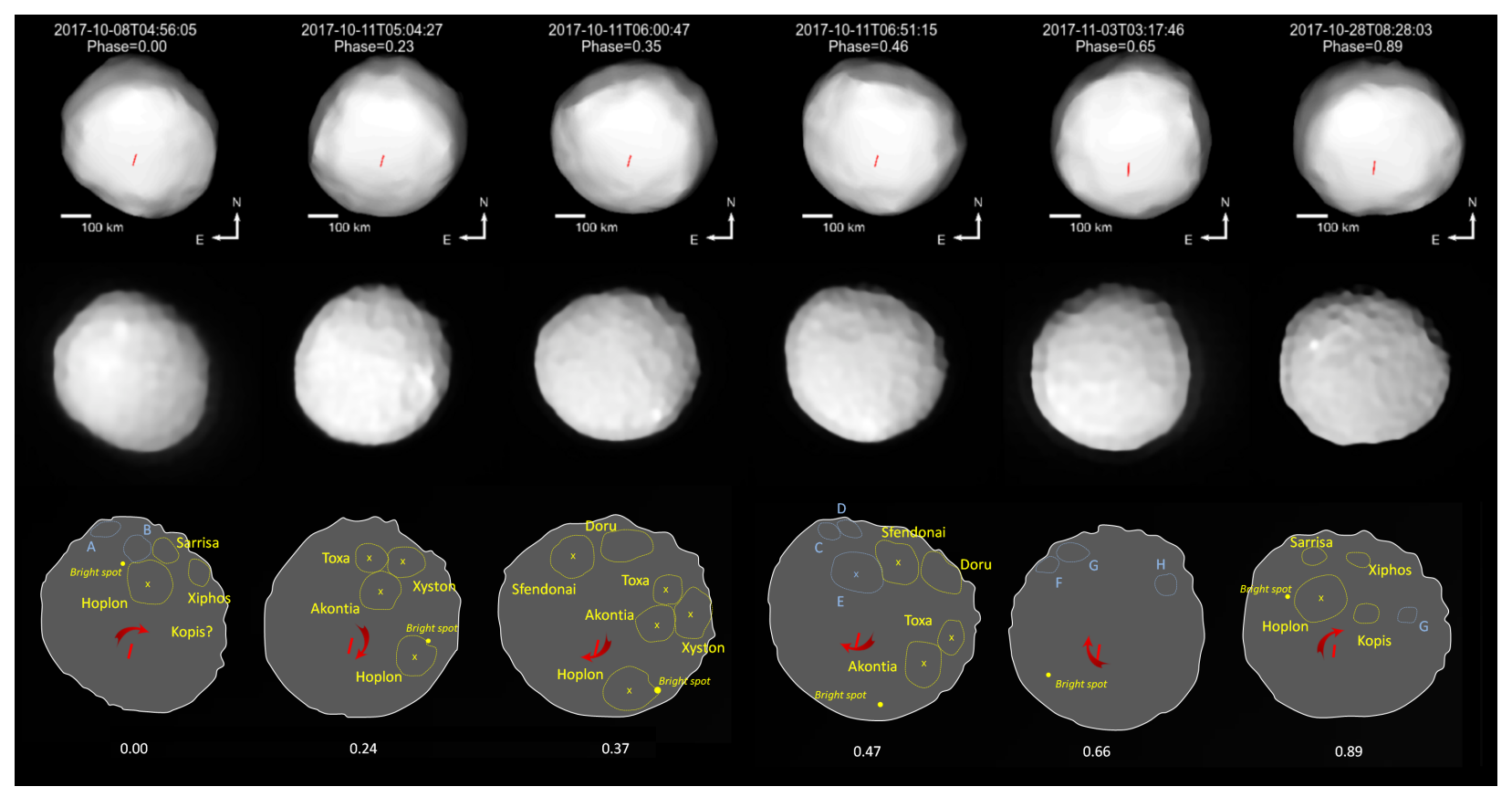

Figure 2: Deconvolved images of (2) Pallas (middle line), compared to projections of the ADAM shape model (top) and sketches highlighting the main geological features identified on Pallas (bottom). The first panel corresponds to the southern hemisphere and the bottom one to its northern hemisphere. Features detected at a single epoch are shown in blue, and those tracked throughout multiple rotation phase angles are in yellow. The epochs are ordered by increasing rotation phase. The red segment indicates Pallas' spin axis. 


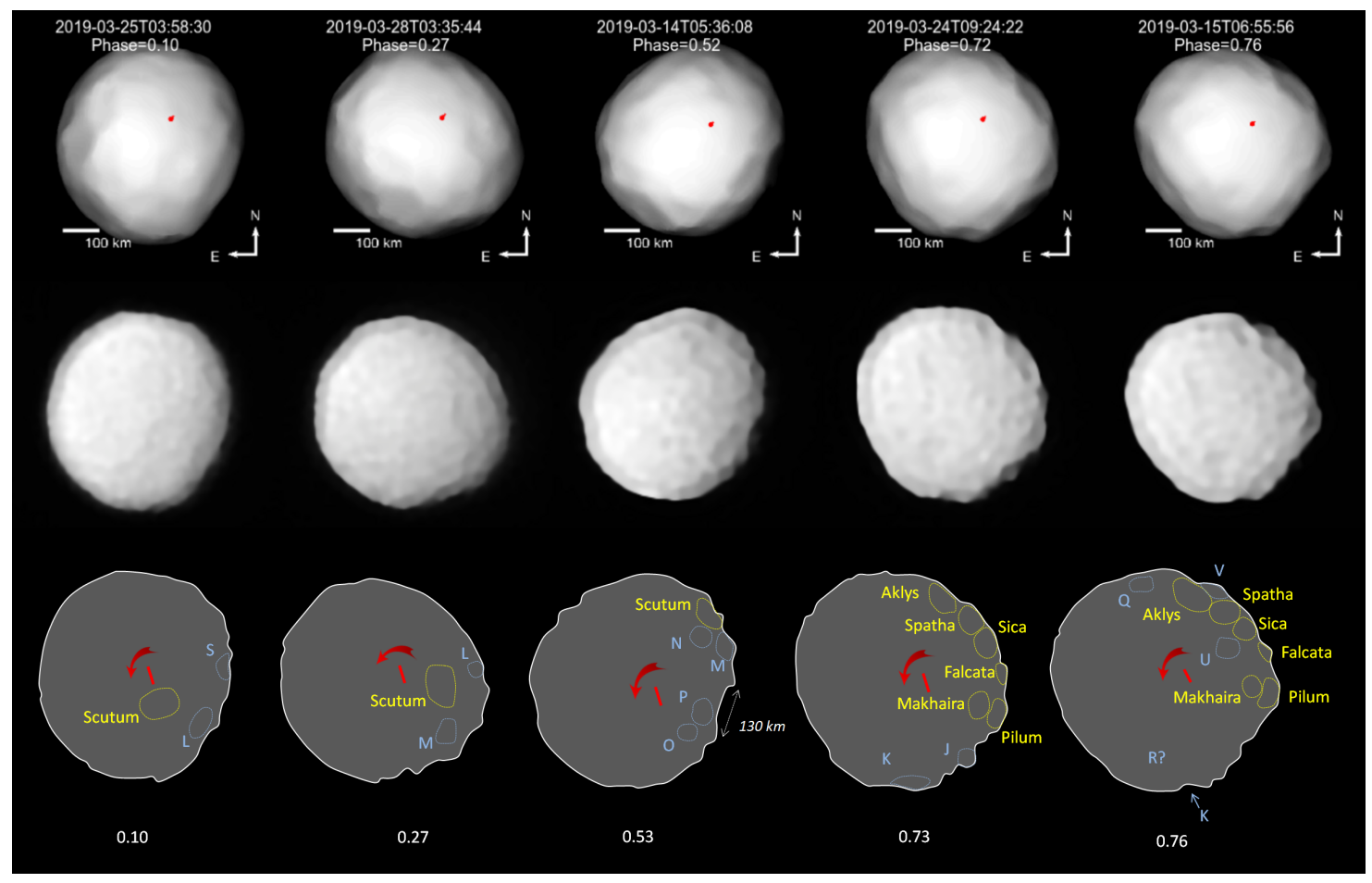

Figure 2: continued. 


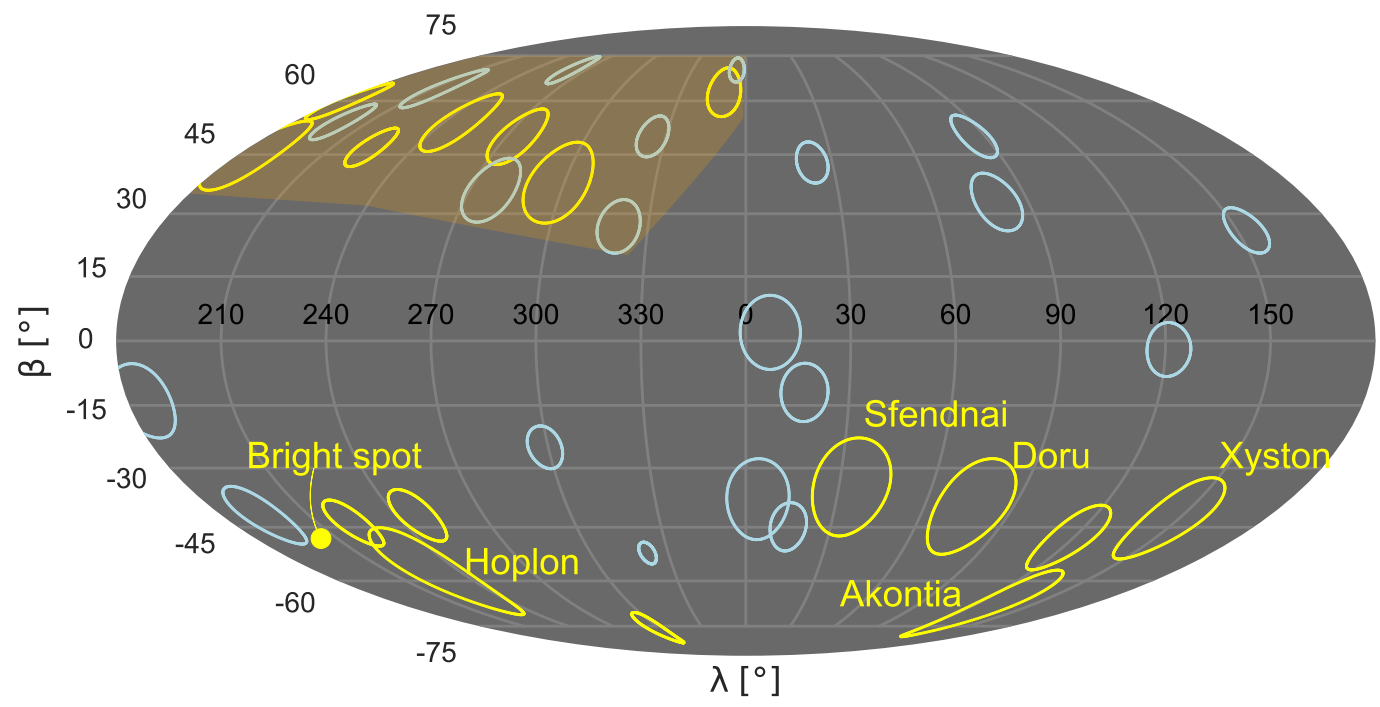

Figure 3: Mollweide projection of the 36 craters and the bright spot identified on the surface of Pallas. The same colour code as in Fig. 2 is used for the craters. The highly cratered region is highlighted in light orange. The name of the five largest craters is indicated. 


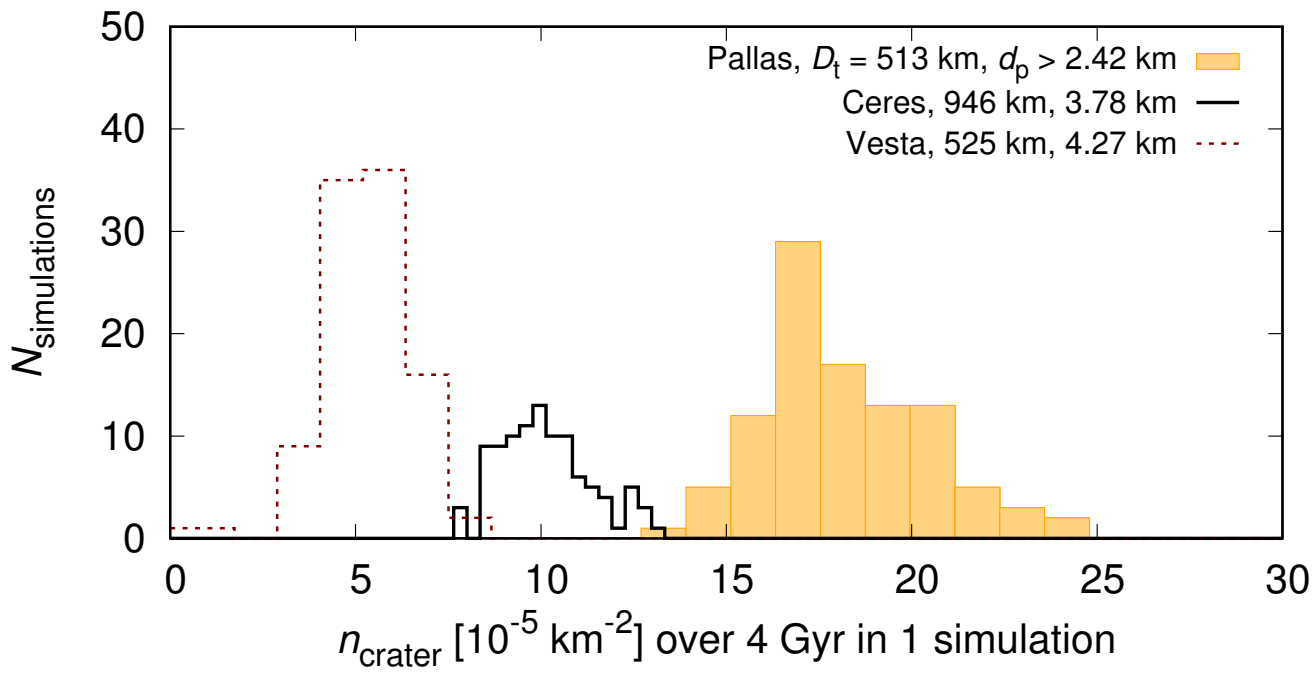

Figure 4: N-body simulations link the heavily cratered surface of Pallas to its highly inclined and eccentric orbit inducing high average impact speed on this body. The histograms show the number of collisional events per surface unit able to create a crater with $D_{\mathrm{c}} \geq 40 \mathrm{~km}$ for each of the three largest asteroids: (1) Ceres, (4) Vesta, and (2) Pallas. A total of 100 Monte-Carlo simulations were ran for each object. The target size $D_{\mathrm{t}}$ and the projectile diameter $d_{\mathrm{p}}$ needed to create the craters are provided in the legend. The high impact rate per surface unit on Pallas reflects its large median impact velocity of $v_{\text {imp }}=11.5 \mathrm{~km} \mathrm{~s}^{-1}$, compared to the typical velocity of $\sim 5.8 \mathrm{~km} \mathrm{~s}^{-1}$ for the main belt. 


\section{Methods}

Cratering on Pallas. Pallas exhibits numerous large $\left(D_{\mathrm{c}}>30 \mathrm{~km}\right)$ impact features, including various complex craters showing evidence for a central peak (Supplementary Fig. 1). The nearly pole-on orientation of the asteroid during our 11 sets of SPHERE observations - 6 for the southern hemisphere and 5 for the northern one - allowed to accurately identify the craters on both hemispheres throughout a complete rotational phase period.

We first removed the brightness gradient from each SPHERE image, which depends on the local illumination (local incidence, reflection and phase angle), following the method outlined in Fétick et al (2019) ${ }^{13}$. The craters were then visually searched on the images, simultaneously using a projection of the ADAM shape model to measure their planetocentric coordinates. Owing to imperfect $\mathrm{AO}$ corrections and deconvolution of the images, many features that could be interpreted as craters might actually be artefacts and/or correlated noise in the images. To avoid false positives, each series of observation was carefully examined, and only features consistently present across a full set of images were recorded. Specifically, each sequence of SPHERE observations consisted in 5 images being simultaneously recorded by the two ZIMPOL cameras ${ }^{9}$, resulting in a total set of 10 images per observing epoch. Confirmed craters are those found throughout at least one complete sequence of 10 images.

We then measured the crater diameter by extracting their brightness profile on the image. We defined the crater edge as the location where the profile inflects symmetrically on both sides of the centre of the crater. Diameters were estimated as the distance between the two opposite ends of 
the edge, orthogonally to the direction of the sub-solar point to account for the viewing angle. For those craters that are visible at multiple epochs of observation, we checked the consistency of our method by comparing values computed from the different epochs.

Using this method, a total of 36 craters were identified on Pallas, including 34 with diameter $D_{\mathrm{c}} \geq 40 \mathrm{~km}$ (Supplementary Table 1). Considering our careful rejection of possible false positives, which probably led to the rejection of a few true features, this number should be considered as a lower limit. In addition, several craters located near the sub-solar point, where shadowing was minimal, might have been also missed. Anyhow, using our volume-equivalent diameter of $D=$ $513 \mathrm{~km}$, the number of 34 craters translates to an observed average number density of $\mathrm{N}\left(D_{\mathrm{c}} \geq\right.$ $40 \mathrm{~km})=4.1-5.5 \times 10^{-5} \mathrm{~km}^{-2}$, i.e., more than twice larger than the average crater density on Vesta in this size range ${ }^{35}$. The interval of values provided here reflects the uncertainty on the surface of Pallas properly sampled by SPHERE: while the full surface was covered, the equatorial region was seen almost edge-on owing to the nearly pole-on orientation of Pallas during our observations. This likely explains the apparent lack of craters located between planetocentric latitudes of -15 and $+15^{\circ}$ (Fig. 3). This region representing $\sim 25 \%$ of the total surface of Pallas, we assumed that between $75-100 \%$ of the surface was accurately covered by our observations, and propagated this assumption to the uncertainty on crater density. Global crater frequency measurements however do not make a lot of sense, because they average crater counts over multiple geological units with different ages. The most heavily cratered area of Pallas is found in the north-west region, between approximately $\lambda=180-0^{\circ}, \beta=35-75^{\circ}$ (Fig. 3). This region represents an area of 7.2 to $9.3 \times 10^{4} \mathrm{~km}^{2}$ and contains 13 craters larger than $40 \mathrm{~km}$ in diameter (14 larger than $30 \mathrm{~km}$ ), implying a crater 
number density $\mathrm{N}\left(D_{\mathrm{c}} \geq 40 \mathrm{~km}\right)=1.6 \pm 0.2 \times 10^{-4} \mathrm{~km}^{-2}$. This is comparable to the oldest and most heavily cratered terrains (HCT) found on Ceres and Vesta, such as the cratered terrain of Ceres's Ezinu quadrangle, with $\mathrm{N}\left(D_{\mathrm{c}} \geq 45 \mathrm{~km}\right)=1.4 \times 10^{-4} \mathrm{~km}^{-2}, 14$ and the North pole of Vesta, with $\mathrm{N}\left(D_{\mathrm{c}} \geq 40 \mathrm{~km}\right)=1.5 \times 10^{-4} \mathrm{~km}^{-2} \stackrel{15}{15}$ The vast majority of geological units on Ceres and Vesta are far less cratered $\sqrt{14+[16}[36,[38$.

Modelled cratering record. To understand the origin of the heavily cratered surface of Pallas, we explored its 4-Ga-long collisional evolution, as well as that of (1) Ceres and (4) Vesta, through series of Monte-Carlo simulations performed with the Boulder code ${ }^{39}[40$. The expected crater density on the three objects was evaluated by extracting all relevant collisional events in an extended set of 100 simulations per object. Specifically, using the $\pi$-scaling ${ }^{17}$ for the relation $D_{\mathrm{c}}\left(d_{\mathrm{p}}\right)$ between the crater and projectile sizes, we recorded all events able to produce $D_{\mathrm{c}} \geq 40 \mathrm{~km}$ in order to allow a direct comparison between simulations and observations. The projectile size needed to create a given crater size and, therefore, the frequency of large collisions in our simulations highly depends on the choice of the scaling law 41 . However, the resulting relative differences between the three bodies (Ceres, Vesta and Pallas) is likely minor when using the same scaling law for all of them.

Collisional probabilities $\left(P_{\mathrm{i}}\right)$ and impact velocities $\left(v_{\mathrm{imp}}\right)$ were computed from the observed orbital distribution of the main belt, and an evolving size-frequency distribution (SFD) providing the best match to the observed SFD after $4 \mathrm{Ga}$ evolution. Relevant input parameters of our simulations were computed from the current osculating orbital elements of the asteroids and are 
summarised in Supplementary Table 5. Using proper orbital elements instead of the osculating ones does not change significantly the value of the derived parameters. For instance, in the case of Pallas, we derived $P_{\mathrm{i}}=2.17 \times 10^{-18} \mathrm{~km}^{-2} \mathrm{a}^{-1}$ and $v_{\mathrm{imp}}=11.49 \mathrm{~km} / \mathrm{s}$ when using current elements $\left(\mathrm{e}=0.23, \mathrm{i}=34.8^{\circ}\right)$, and $P_{\mathrm{i}}=1.89 \times 10^{-18} \mathrm{~km}^{-2} \mathrm{a}^{-1}$ and $v_{\mathrm{imp}}=11.25 \mathrm{~km} / \mathrm{s}$ for proper elements $\left(e=0.28, i=33.2^{\circ}\right)$, implying a variation of $\sim 10 \%$ and $\sim 2 \%$, respectively. The resulting synthetic crater densities for Ceres, Vesta and Pallas are as followed: $10 \pm 3 \times 10^{-5} \mathrm{~km}^{-2}$, $6 \pm 3 \times 10^{-5} \mathrm{~km}^{-2}$, and $19 \pm 5 \times 10^{-5} \mathrm{~km}^{-2}$, respectively (Fig. 4). Here, the range of values reflect the Poisson uncertainty due to the stochasticity of the collisional process.

The derived estimates directly relate to the different collisional environment of the three objects. In particular, Pallas is located in a more violent environment due to its eccentric $(e=0.23)$ and highly-inclined $\left(i=34.8^{\circ}\right)$ orbit that implies substantially larger impact velocities $v_{\text {imp }}$. This, of course, increases the number of available projectiles, because $d_{\mathrm{p}}$ needed to create $D_{\mathrm{c}} \geq 40 \mathrm{~km}$ is smaller, and the size frequency distribution (SFD) of the asteroid belt is steep (slope -2.5 in this size range $\left.\mathrm{e}^{(18}\right)$. This is only partially compensated by the lower intrinsic probability of collisions between Pallas and impactors from the asteroid belt. Ceres is about twice larger than the other two bodies but gravitational focussing, expressed as $f_{\mathrm{g}}=1+\left(v_{\mathrm{esc}} / v_{\mathrm{imp}}\right)^{2} \doteq 1.01$, where $v_{\mathrm{esc}}$ is the escape velocity and $v_{\text {imp }}$ the impact velocity, does not contribute significantly.

3D shape reconstruction. We used the All-Data Asteroid Modeling (ADAM) inversion procedure $19,42,45$ to reconstruct the shape and spin of Pallas, using as input the complete set of disk-resolved images and optical lightcurves listed in Supplementary Tables 2 and 3, respectively, and occultation 
data described in Hanuš et al. (2017) ${ }^{45}$ for sanity checks. Our set of images comprises both our VLT/SPHERE observations, as well as Keck/NIRC2 images retrieved from the Keck Observatory Archive (KOA). While the NIRC2 images have a lower angular resolution than the SPHERE ones, these images sample additional observing geometries of Pallas that are complementary to our own dataset for shape reconstruction. We first created a low-resolution shape model using the spherical harmonics parameterization and our complete dataset of images and light curves as input. Due to the disparity in imaging resolution of the Keck/NIRC2 $2^{46}$ and VLT/SPHERE ${ }^{599}$ images, we then constructed a higher resolution model from the SPHERE data only, using the low-resolution shape model as initial input. Finally, we allowed the vertices to move independently of parameterization, subject only to the regularization and AO data fit functions.

Overall, our model fits the image boundaries at the sub-pixel level and recovers most of the high-resolution features present in the SPHERE images (Fig.2). The best triaxial ellipsoid fit to the $3 \mathrm{D}$-shape model has a volume-equivalent diameter of $\mathrm{D}=513 \pm 6 \mathrm{~km}$, with semi-axes along the principal axes of inertia $(284 \times 266 \times 224) \pm 6 \mathrm{~km}$. An equator-on projection of the model reveals that the South Pole is substantially flatten (Supplementary Fig. 3), which could relate to the existence of a large basin, possibly created by a single or a few significant impacts, like Rheasilvia on Vesta ${ }^{20}$. This feature is unseen on the SPHERE images, due to the nearly pole-on orientation of the asteroid during the observations, and could only be retrieved thanks to the 3D-shape reconstruction and the use of complementary light curves. The basin would represent $\sim 6 \pm 1 \%$ of the current volume of Pallas. Its polar location is consistent with reorientation of the rotation axis towards maximum moment of inertia, which occurs over timescales of the order of $\sim 10^{5}$ a for a Pallas-size body 4. 
The fossile shape of Pallas. Our ADAM shape model was further used to investigate the hydrostatic shape of (2) Pallas, assuming both an homogeneous and a two-layer differentiated interior. The hydrostatic equilibrium figure of a homogeneous body can be computed using the MacLaurin equation, whereas for a differentiated body it requires to be solved numerically. Here, we used a numerical integration of the Clairaut's equations developed to an order that depends on the geodetic parameter $m^{48 / 49}$ that is function of the angular spin velocity and mean density of the body. Depending on the value of that parameter and the accuracy of available observations, the Clairaut's equations may be developed to first, second or third order ${ }^{50}$. This method has been previously applied to the hydrostatic figures of the Earth ${ }^{48}$ and Ceres ${ }^{2149}$.

Supplementary Fig. 2 compares the (a-c) dimension of Pallas with respect to a similar-size body at equilibrium, where a and $\mathrm{c}$ are the equatorial and polar radii of the object. The shape of Pallas significantly deviates from equilibrium considering its current rotation period $(\sim 7.8 \mathrm{~h})$, implying it was significantly reshaped by a large impact, and/or that it used to rotate faster in the past. We investigated whether the putative South Pole basin could account for this deviation. To do so, a best-fit ellipsoid was adjusted to the 3D-shape model of Pallas, excluding the South Pole (specifically, meshes below $-31^{\circ}$ latitudes were rejected from the fit; Supplementary Fig. 3). The resulting ellipsoid has semi-major axis $282 \times 262 \times 249 \mathrm{~km}$, which is closer to an hydrostatic shape, but still requires a change of rotation period of $1.6 \mathrm{~h}$, down to $\sim 6.2 \mathrm{~h}$, to be at equilibrium.

For a homogeneous sphere, the change in angular momentum is given by $\Delta L=2 / 5 M R^{2} 2 \pi / \mid P-$ $P^{\prime}$, where $M$ is the mass, $R$ the radius and $P$ the rotation period. If we simply assume $\Delta L=$ 
$m_{\mathrm{p}} v_{\text {imp }} \sin \left(45^{\circ}\right) R$, where subscript $\mathrm{p}$ refers to 'projectile', and express $d_{\mathrm{p}}=2 \times\left[3 m_{\mathrm{p}} /\left(4 \pi \rho_{\mathrm{p}}\right)\right]^{1 / 3}$, then the projectile size needed for $\left|P-P^{\prime}\right|=1.6 \mathrm{~h}$ is $d_{\mathrm{p}} \approx 48 \mathrm{~km}$ assuming $\rho_{\mathrm{p}}=3000 \mathrm{~kg} \mathrm{~m}^{-3}$ and $v_{\mathrm{imp}}=11.5 \mathrm{~km} / \mathrm{s}$. This translates to a crater size of $\sim 370 \mathrm{~km}$ according to the $\pi$-scaling law $\frac{117}{\text {, i.e., }}$ $\sim 70 \%$ of the current size of Pallas, which could represent the South-pole basin. Therefore, it seems very plausible that the fitted ellipsoid in Supplementary Fig. 3 represents the original, pre-impact shape of Pallas.

The present-day Pallas family. Pallas is surrounded by a few hundreds small $(\mathrm{D}<20 \mathrm{~km}-$ sized) bodies that together form a distinct asteroid family ${ }^{51}$. We describe here the method we used to identify the family members, whose orbital properties were subsequently used to examine the physical conditions of the family forming event.

The Pallas family is well-defined and taxonomically homogeneous. It is located at high inclination, where few background asteroids exist. The geometric albedo of the family members is comprised in the range $p_{V} \in(0.06 ; 0.24)^{\sqrt[52]{2}}$, and colours from the SDSS $S^{53}$ are such that the colour index $a^{\star}<0$ mag. The dynamic environment of Pallas is complex and affected by several meanmotion and secular resonances. As a consequence, many asteroids are on chaotic, unstable or resonant orbits, with proper orbital elements that can quickly shift in eccentricity and/or inclination. Because of this, many family members can be missed when identifying them using proper elements. We therefore instead chose to consider the averaged mean orbital elements of the asteroids, including all forced terms. The mean elements were computed carefully to avoid aliasing of fast orbital frequencies, with a four-stage convolution filter based on the Kaiser windows ${ }^{54}$. Input 
sampling of the osculating elements was set to 1 year, and we used four filters denoted A, A, A, B with decimation factors $10,10,5,3$, resulting in output sampling of the mean elements of 1500 years. Finally, we applied a running-average filter with window $1 \mathrm{Ma}$ and output sampling $0.1 \mathrm{Ma}$. Using this method, we were able to use all of the observed multi-opposition asteroids, not only those with stable proper elements (473 vs 319 bodies). We used exactly the same algorithm for our synthetic families generated for investigating the orbital evolution of the Pallas family through $\mathrm{N}$ body simulations (see below), allowing a direct comparison of our simulations with observations. Supplementary Fig. 6 displays the mean orbital elements of the observed population of the family members, interlopers and background asteroids in the vicinity of Pallas.

Orbital evolution of the family. The long-term orbital evolution of the Pallas family was studied by use of the symplectic N-body integrator Swift-Rmvs $3^{55}$ in order to estimate the age of the family. We explored the simplest case in which the current family was created in a single large collision. More complicated scenarios, e.g., in which the family was subsequently rejuvenated by smaller impacts, are beyond the scope of this work. Our dynamical mode ${ }^{56}$ included the outer solar system planets, and a barycentric correction to account for the inner planets. Pallas was treated as a massive body, as close encounters can enhance diffusion in its vicinity. Our code further included the Yarkovsky diurnal and seasonal effects 5758 , the YORP effect ${ }^{59}$, and reorientation or reshaping during random collisions and when bodies reach a critical spin rate. The time step was set to $\Delta t=36.525 \mathrm{~d}$, and the time spanned up to $4 \mathrm{Ga}$.

We created a synthetic family of 1380 bodies with assumed isotropic velocities ${ }^{60}$ and spins, 
escape velocity $v_{\mathrm{esc}}=324 \mathrm{~m} \mathrm{~s}^{-1}$, and maximum velocity difference $v_{\max }=600 \mathrm{~m} \mathrm{~s}^{-1}$. We derived the preferred true anomaly $f=140^{\circ}$ and argument of perihelion $\omega=60$ at the time of impact, using the Gauss equations to fit ellipses to the distribution of the Pallas family in the $a_{\mathrm{m}}$ vs $e_{\mathrm{m}}$ and $a_{\mathrm{m}}$ vs $\sin I_{\mathrm{m}}$ spaces of the mean orbital elements.

Thermal parameters of our model included the bulk density $\rho=2890 \mathrm{~kg} \mathrm{~m}^{-3}$, the density of surface layers (regolith) $\rho_{\text {surf }}=1500 \mathrm{~kg} \mathrm{~m}^{-3}$, the heat capacity $C=680 \mathrm{~J} \mathrm{~kg}^{-1} \mathrm{~K}^{-1}$, the thermal conductivity $K=10^{-3} \mathrm{~W} \mathrm{~m}^{-1} \mathrm{~K}^{-1}$, the Bond albedo $A=0.10$, and the infrared emissivity $\epsilon=0.9$. When unknown, diameters $D$ were computed from absolute magnitudes $H$ assuming the median value of geometric albedo of the Pallas family members $p_{V}=0.122^{61}$.

In order to compare our N-body integration with the observed family, we used the method of Broz \& Morbidelli (2019) $)^{62}$, which consists in rescaling the synthetic population to match the observed SFD, and then computing the $\chi^{2}$ for the number of objects counted in boxes defined in the $\left(a_{\mathrm{m}}, e_{\mathrm{m}}\right)$ space (Supplementary Fig. 7). The evolution of the $\chi^{2}(t)$ throughout our integration is shown in Supplementary Fig. 8. It decreases from the initial value $\chi^{2} / N_{\text {box }} \simeq 3.6$ down to 1.35 , with the best-fit value corresponding to $t=1.68 \mathrm{Ga}$. The uncertainty on the age was computed from the scatter of the $\chi^{2}(t)$ values due to the random selection procedure, assuming the best-fit value is acceptable. By doing so, we derived an estimate of the family's age of $t=1.3$ to $1.9 \mathrm{Ga}$.

As a by-product, we computed the exponential decay time scales for bodies of various sizes, which are necessary inputs for Monte-Carlo collisional models (see below). The values are $\tau=$ $374,419,782,1390,2050$, and $2130 \mathrm{Ma}$ for the size bins between $D=0.5,1,2,5,10,20,30 \mathrm{~km}$, 
respectively.

The family-forming impact. We then performed combined SPH/N-body simulations ${ }^{63}+65$ aiming at deriving the impact parameters providing the best fit to the orbital distribution and SFD of the Pallas family members, and allowing a direct comparison of the resulting impact features with observations. Here again, we assumed that the Pallas family originated from a single large collision. While multiple small events may eject enough material to produce the present-day family, a large impact is needed to account for the size of the largest observed fragment $\left(D_{\mathrm{lf}} \sim 20 \mathrm{~km}\right)$. In addition, fragments produced in a cratering event are usually smaller than the projectile, and km-size fragments are continuously removed from the family by Yarkovsky $\operatorname{drift}^{57 \mid 58}$ and chaotic diffusion over timescales of a few hundred million years, implying they cannot accumulate over $4 \mathrm{Ga}$. Consequently, our simulations, which are constrained by $\mathrm{D}>10 \mathrm{~km}$ fragments, are not affected by small cratering events.

Our model included a fragmentation without gravity $\sqrt{66}$ and gravitational reaccumulation ${ }^{67}$. We used Tillotson (1962)'s equation of state ${ }^{68}$, vonMises (1913)'s yielding criterion ${ }^{69}$, and Grady \& Kipp (1980)'s fracture model ${ }^{70}$. Initial conditions included two spherical bodies (the target and the projectile), with target size $D_{\mathrm{pb}}=513 \mathrm{~km}$, and impact velocity $v_{\mathrm{imp}}=12 \mathrm{~km} \mathrm{~s}^{-1}$. Our simulations covered a range of specific energy ratios $Q / Q_{\mathrm{D}}^{\star}$, where $Q_{\mathrm{D}}^{\star}$ denotes the strength from the scaling law, provided in Supplementary Table 6. We used an SPH discretisation in space, with number of particles $N_{\text {part }} \doteq 1.4 \times 10^{5}$, and a predictor-corrector discretisation in time. The time step was limited by the Courant criterion, and to limit changes in energy, pressure and fracture 
damage per time step to accurately control the integrations as described in Benz \& Asphaug (1994, $1995) \longdiv { 6 6 7 1 }$. Given the target size and $v_{\text {imp }}$, the chosen time span was $200 \mathrm{~s}$. We used standard artificial viscosity parameters $\alpha_{\mathrm{av}}=1.5, \beta_{\mathrm{av}}=3.0$, and a modification of the scalar damage $\mathcal{D}$, as in Ševeček et al. (2017) ${ }^{65}$. Concerning the N-body part of the simulation, we used a handoff relation $R_{i}=\left[3 m_{i} /\left(4 \pi \rho_{i}\right)\right]^{1 / 3}$, a tree-code with the opening angle $\theta=0.5 \mathrm{rad}$, and a hexadecapole approximation for the gravity. We assumed a perfect merging. The time step was $\Delta t=10^{-6}$ (in $G=1$ units), and the time span $50000 \Delta t$.

Our simulations covered a relevant range of outcomes, shown in Supplementary Fig. 9, from weakly catastrophic to large cratering events. In every simulations the target was fully damaged. The velocity field at the end of the fragmentation phase indicates that the first three higher-energy impacts $Q / Q_{\mathrm{D}}^{\star} \geq 0.067$ affected essentially the whole surface of the target, while the last three produced a large crater and only partial modification of the surface.

We determined the excavated mass before fall-back as the sum of all particles located above $r>R+30 \mathrm{~km}$, allowing for some expansion of the target. The simulations that better match the observed SFD of the family (rows 3 and 4 in Supplementary Table 6) have excavated mass $M_{\mathrm{ex}}=0.016$ to 0.027 (in $M_{\mathrm{pb}}$ units), implying the equatorial excavation is more likely to be linked to the present-day Pallas family than the South-pole basin. From Supplementary Fig. 9, we measured a transient crater size of at least $250 \mathrm{~km}$, which can subsequently increase, possibly up to the target size, during relaxation of the surface. However, the crater may not be well-preserved in the highest-energy impacts due to substantial reaccumulation. 
Supplementary Fig. 10 shows the SFD of the fragments after reaccumulation, assuming their final density is the same as before the impact $\left(\rho_{0}=2.89 \mathrm{~g} \mathrm{~cm}^{-3}\right)$. If we assume that the ejected fragments have retained their expanded densities, $\rho<\rho_{0}$, this would shift their SFD toward slightly larger $D$, possibly by a factor of 1.5 . Consequently, lower-energy oblique impacts would produce a better fit to the observed SFD. Our simulations covered a reasonable range of $Q / Q_{\mathrm{D}}^{\star}$, so that the largest fragments have sizes $D_{\mathrm{lf}}=14$ to $36 \mathrm{~km}$ that are relatively close to the observed value $\left(D_{\mathrm{lf}}=22.46 \mathrm{~km}\right)$. The synthetic SFDs have significantly steeper slope than the observed one (approximately -5.0 vs -2.2 ), which indicates significant subsequent collisional and orbital evolution.

Next, the ejected mass was estimated as the sum of all fragments from the target. We did not include the projectile, which either vaporized, or whose remaining fragment escape the space of proper elements of the family. Ejected masses are comprised between 0.015 to 0.028 ( $M_{\mathrm{pb}}$ units; Supplementary Table 6), which is comparable to $M_{\mathrm{ex}}$.

\section{Evolution of the Size-Frequency Distribution of the family. Independent constraints on} the age of the family were derived using a Monte-Carlo collisional model, using as input the synthetic SFDs derived from our SPH simulations for the initial family. This method simultaneously allows to estimate the probability that such a family is created over the course of evolution. We assumed constant intrinsic collisional probabilities $P_{\mathrm{i}}=2.86 \times 10^{-18}, 2.17 \times 10^{-18}$, and $2.87 \times 10^{-18} \mathrm{~km}^{-2} \mathrm{a}^{-1}$ for the three relevant combinations of collisions (MB-MB, MB-Pallas, Pallas-Pallas), and mutual impact velocities $v_{\mathrm{imp}}=5.77,11.49$, and $13.05 \mathrm{~km} \mathrm{~s}^{-1}$, computed ac- 
cording to Bottke \& Greenberg $(1993)^{72}$. Our model includes a size-dependent dynamical decay from Bottke et al. $(2005)^{73}$ for the main belt, and from our previous N-body simulation for the Pallas family.

A number of additional parameters were specified, including the scaling law $Q_{\mathrm{D}}^{\star}(r)$, which was taken from Benz \& Asphaug (1999) $)^{74}$ for basalt material, with $\rho=2.89 \mathrm{~g} \mathrm{~cm}^{-3}$, at the impact velocity $5 \mathrm{~km} \mathrm{~s}^{-1}$. This is inconsistent with typical velocities on Pallas, but it cannot be easily improved unless a big matrix of simulations is computed. We also used a modification of the parametric relation for the mass of the largest fragment $M_{\mathrm{lf}}\left(Q / Q_{\mathrm{D}}^{\star}\right)$, which seems necessary for small cratering events ${ }^{75}$. Initial conditions are quite close to the observed SFD, except for the synthetic family which is steeper ( -5.0 cumulative). A discretisation in mass is performed with a logarithmic factor 1.5 . The output time step of the simulation was set to $\Delta t=10 \mathrm{Ma}$, and the nominal time spanned $4 \mathrm{Ga}$. At least 10 Monte-Carlo simulations were performed, because of fractional probabilities of large breakups and lower-probability events.

Results are summarised in Supplementary Fig. 11. A typical time scale of a significant (10\%) evolution of the family's SFD is of the order of $100 \mathrm{Ma}$, mostly due to dynamical decay and secondary MB-Pallas collisions. After $2 \mathrm{Ga}$ of evolution, about a third of simulations produced synthetic families with $D>22.46 \mathrm{~km}$ for the largest fragment. We therefore consider the Pallas family to be a likely outcome of the equatorial excavation forming event. The event responsible for the South-pole basin, on the other hand, requires up to 3-to-4 Ga of evolution owing to the steeper SFD of the collisional fragments. This longer time is in contradiction with the simulated orbital 
evolution of the family, implying that the South-pole basin is unlikely to relate to the present-day Pallas family.

Present-day composition of Pallas. Combined with available mass estimates from the literature (Supplementary Table 4 and Supplementary Fig. 4), our 3D-shape model of Pallas returns a bulk density of $2.89 \pm 0.08 \mathrm{~g} / \mathrm{cm}^{3}$, in perfect agreement with the grain density of CM chondrite meteorites $\left(2.90 \pm 0.08 \mathrm{~g} / \mathrm{cm}^{3}\right)^{21}$ assuming near zero porosity in the interior of Pallas. Whereas CM chondrites exhibit the same hydration signature in the $3-\mu \mathrm{m}$ wavelength range as Pallas ${ }^{6}$, these meteorites are usually linked to $\mathrm{Ch} / \mathrm{Cgh}$-type asteroids ${ }^{22 / 76 / 77}$ and have distinct spectral properties from Pallas in the visible and near-infrared $(0.4-2.5 \mu \mathrm{m})$. Specifically, Pallas is bluer and brighter than most $\mathrm{CM}$ chondrites and it does not exhibit the $0.7-0.9-\mu \mathrm{m}$ absorption features that is present in the meteorite spectra.

A direct link between Pallas and CM chondrites therefore does not appear obvious. It is possible, however, that Pallas and the parent bodies of $\mathrm{CM}$ chondrites accreted from the same initial material, as suggested by their similar densities, and that their spectral differences come from distinct subsequent thermal and collisional evolution owing to Pallas' large size and distinct collisional environment. In particular, frequent high-energy impacts and micro-meteorite bombardement on Pallas could have led to partial dehydration of its surface, which could explain its bluer and brighter spectrum and the lack of phyllosilicate signatures in the visible. On the other hand, the 3- $\mu \mathrm{m}$ signature would have been preserved because of its much deeper and broader profile. Along these lines, laboratory experiments have shown that artificially heated CM chondrites 
usually exhibit bluer, brighter (although not as bright as Pallas) and more featureless spectra ${ }^{78} 79$. Based on these considerations, it appears possible that Pallas represents the parent body of heated CM chondrites, for which no parent body has been identified so far.

Pallas' derived bulk density is further higher than Ceres' $\left(2.16 \pm 0.01 \mathrm{~g} / \mathrm{cm}^{3}\right)^{2}$, suggesting a lower water-to-rock ratio, in agreement with its higher and seemingly more stable topography. A lower water content for Pallas with respect to Ceres is also in agreement with the survival of the Pallas family members over several hundred million years, while the lack of a Ceres family points towards rapid sublimation of impact fragments from Ceres ${ }^{80}$.

Initial rock-to-ice ratio of Pallas. Assuming Pallas accreted from a mixture of anhydrous dust and ice, two distinct evolutionary pathways must be considered when assessing its early internal evolution. In the first scenario, Pallas accreted with about the same bulk water content as inferred from its measured density. This leads to a low water-to-rock ratio $(\mathrm{W}: \mathrm{R}<1)$ in the transient ocean generated by the decay of short-lived radioisotopes. In that case, Pallas did not differentiate, and its current surface would represent a collisionally evolved version of its original one. In the alternate case where Pallas' initial W:R was high ( $\gg 1)$, thermophysical modelling predicts the formation of an icy outer shell through the separation of water from the silicates, upward flowing and freezing towards the surface. This icy shell being missing at present implies it would have been progressively removed by collisions exposing fresh ice and thus triggering their sublimation. In that scenario, today's Pallas surface would represent the hydrated mantle of the proto-Pallas.

Considering that the measured density of Pallas is fully compatible with CM chondrites, as 
well as with its predicted mineralogy in the low W:R scenario studied by Castillo-Rogez et al. $(2018)^{81}$, which is also consistent with aqueous alteration conditions inferred for CM chondrites ${ }^{82}$, we favour the low W:R scenario for its formation and evolution. Importantly, the modelled mineralogy includes small fractions of salts (e.g., carbonates, chlorides), the presence of which could explain both the higher albedo of the Pallas family compared to other B-type asteroids $\frac{61883}{\text {, and }}$ albedo variations seen on the surface of Pallas.

Formation time and interior of Pallas. Whether Pallas' internal temperature reached the silicate dehydration threshold ( $\sim 820 \mathrm{~K})$ depends on its time of formation. One-dimensional thermal conduction was modelled using the approach developed by Castillo-Rogez et al. (2007) ${ }^{84}$ and applied to a variety of bodies, including Pallas ${ }^{85}$. Specifically, heat was transferred by conduction with the following equation:

$$
\frac{\partial(k(T) \partial(T) / r)}{\partial r}+\frac{2}{r}\left(k(T) \frac{\partial T(r)}{\partial r}\right)=\rho(r) C_{p}(T) \frac{d T(r)}{d t}-H(r)
$$

where $T$ is temperature (in Kelvin), $r$ local radius, $k$ thermal conductivity, $\rho$ material density, $C_{p}$ specific heat, $t$ time, $H$ internal heating (i.e., radioisotope decay heat). Calculation of the radioisotope decay heat, the main heat source for Pallas, can be found in Supplementary Table 7. The properties of the materials used in the modelling are listed in Supplementary Table 8. Pure serpentine has a thermal conductivity of about $2.5 \mathrm{~W} / \mathrm{m} / \mathrm{K}$ while anhydrous silicates (olivine and pyroxene) have thermal conductivities up to $5 \mathrm{~W} / \mathrm{m} / \mathrm{K}^{86}$. The latter could be present if aqueous 
alteration was partial. Also, the presence of iron-rich compounds in the rock (like iron sulfide and oxides) could increase the thermal conductivity further. In this study, we covered a range of thermal conductivities for the mantle from 0.5 to $2.5 \mathrm{~W} / \mathrm{m} / \mathrm{K}$ under the assumption that aqueous alteration might be advanced.

Using this model, we found that partial dehydration of the core of Pallas occurs for times of formation $\mathrm{T}_{0}<2.5 \mathrm{Ma}$ after the formation of CAIs (Supplementary Fig. 5). Provided that the proposed association between CM chondrite meteorites and Pallas is correct, and considering the isotopic ages of $\mathrm{CM}$ chondrites (mostly $>3.0 \mathrm{Ma}$ after the formation of CAIs) ${ }^{\sqrt{23}}$, we conclude that the amount of radioisotopes accreted by Pallas was too low to trigger large-scale silicate dehydration and the differentiation of a denser silicate core below a hydrated mantle, thus implying a rather homogeneous interior. This finding is consistent with previous studies that found the primordial internal structure of CM parent bodies to be globally homogeneous $22[87.89$. However, considering Pallas' large size, early partial differentiation (water separation and upward flow) must have occurred in its interior and could explain the high albedo and its variations by an enrichment in salts through aqueous alteration.

The presence of salts in Pallas would further provide a natural explanation to the diversity of sodium contents measured in the Geminids meteor stream 28 . The Geminids are believed to originate from the 5-6-km Apollo-type asteroid (3200) Phaethon ${ }^{33}$, a proposed fragment from the Pallas family that would have been emplaced in the near-Earth space following gravitational interactions with the Jovian mean-motion resonances ${ }^{26,27}$. The proposed link between Pallas and 
Phaethon, however, remains matter of debate: while the spectra of Phaethon, Pallas, and the Pallas family members are strikingly similar in the visible and near-infrared, estimates of the albedo of Phaethon based on thermal measurements show some controversy: some values are consistent with those derived for Pallas and the Pallas family members ${ }^{90}=2$, while others are significantly lower ${ }^{93}$. Polarimetric studies provide an independent insight into this controversy as the albedo of an asteroid can be evaluated from its maximum value of linear polarization degree $\mathrm{P}_{\max }{ }^{94}$ and/or from its polarimetric slope $h$ 95]96. High $\mathrm{P}_{\max }$ values usually correspond to low albedos that are typical of C-type asteroids ${ }^{94,97}$. In case of Phaethon, however, the high value of $\mathrm{P}_{\max } \operatorname{might}$ be better explained by a large average regolith grain size and perhaps also a large surface porosity ${ }^{94}$. Albedo estimates derived from the polarimetric slope $h$, on the other hand, are less dependent on particle size. In the case of Phaethon, the albedo derived from the $h$ value is intermediate $(14 \pm 4 \%)^{98}$, in agreement with Pallas.

Finally, it should be noted that Phaethon does not exhibit the 3- $\mu \mathrm{m}$ absorption band that characterises Pallas ${ }^{99}$. Whether this difference is due to the thermal evolution of Phaethon's surface (e.g., the complete dehydration of surface minerals) in the near-Earth space, or to the fact that Phaethon is not genetically linked to Pallas, remains an open question that should be addressed by the future DESTINY + fly-by mission to Phaethon ${ }^{100}$ or by acquiring high-quality mid-infrared spectra of both Pallas and Phaethon with the James-Webb Space telescope.

Data availability. As soon as papers for our large program are accepted for publication, we make the corresponding reduced and deconvolved AO images and 3D shape models publicly available 
at http: //observations. lam. fr/astero//

Code availability. The code used to generate the 3D shape is available at https://github . com/ matvii/ADAM. The modified SWIFT integrator used to model the orbital evolution of the Pallas family is available at http://sirrah.troja.mff.cuni.cz/ mira/mp/.

34. Liu, Z. et al. A global database and statistical analyses of (4) Vesta craters. Icarus 311, 242-257 (2018).

35. Marchi, S. et al. The Violent Collisional History of Asteroid 4 Vesta. Science 336, 690 (2012).

36. Marchi, S. et al. The missing large impact craters on Ceres. Nat. Commun. 7, 12257 (2016).

37. Pasckert, J. H. et al. Geologic mapping of the Ac-2 Coniraya quadrangle of Ceres from NASA's Dawn mission: Implications for a heterogeneously composed crust. Icarus 316, 2845 (2018).

38. Morbidelli, A., Bottke, W. F., Nesvorný, D. \& Levison, H. F. Asteroids were born big. Icarus 204, 558-573 (2009). 0907.2512.

39. Cibulková, H., Brož, M. \& Benavidez, P. G. A six-part collisional model of the main asteroid belt. Icarus 241, 358-372 (2014). 1407.6143.

40. Werner, S. C. \& Ivanov, B. A. Exogenic Dynamics, Cratering and Surface Ages. In Treatise on Geophysics, 2nd Edition, 327-365 (Elsevier, 2015). 
41. Viikinkoski, M. et al. VLT/SPHERE- and ALMA-based shape reconstruction of asteroid (3) Juno. Astron. Astrophys. 581, L3 (2015).

42. Viikinkoski, M. Shape Reconstruction from Generalized Projections. Ph.D. thesis, Tampere University of Technology (2016).

43. Marsset, M. et al. 3D shape of asteroid (6) Hebe from VLT/SPHERE imaging: Implications for the origin of ordinary H chondrites. Astron. Astrophys. 604, A64 (2017).

44. Hanuš, J., Marchis, F., Viikinkoski, M., Yang, B. \& Kaasalainen, M. Shape model of asteroid (130) Elektra from optical photometry and disk-resolved images from VLT/SPHERE and Nirc2/Keck. Astron. Astrophys. 599, A36 (2017).

45. McLean, I. S. \& Chaffee, F. H. Instrum. for the Keck Observatory. In Iye, M. \& Moorwood, A. F. (eds.) Optical and IR Telescope Instrum. and Detectors, vol. 4008 of Proc. SPIE, 2-7 (2000).

46. Burns, J. A. \& Safronov, V. S. Asteroid nutation angles. Mon. Not. R. Astron. Soc. 165, 403 (1973).

47. Chambat, F., Ricard, Y. \& Valette, B. Flattening of the Earth: further from hydrostaticity than previously estimated. Geophys. J. Int. 183, 727-732 (2010).

48. Rambaux, N., Chambat, F. \& Castillo-Rogez, J. C. Third-order development of shape, gravity, and moment of inertia for highly flattened celestial bodies. Application to Ceres. Astron. Astrophys. 584, A127 (2015). 
49. Lanzano, P. The Equilibrium of a Rotating Body of Arbitrary Density. Astrophys. Space Sci. 29, 161-178 (1974).

50. Nesvorný, D., Brož, M. \& Carruba, V. Identification and Dynamical Properties of Asteroid Families. In Asteroids IV, 297-321 (University of Arizona Press, Tucson, 2015).

51. Mainzer, A. K. et al. NEOWISE Diameters and Albedos V1.0. NASA Planet. Data Syst. 247, EAR-A-COMPIL-5-NEOWISEDIAM-V1.0 (2016).

52. Ivezić, Ž. et al. Color Confirmation of Asteroid Families. Astron. J. 124, 2943-2948 (2002). astro-ph/0208098.

53. Quinn, T. R., Tremaine, S. \& Duncan, M. A three million year integration of the earth's orbit. Astron. J. 101, 2287-2305 (1991).

54. Levison, H. F. \& Duncan, M. J. The long-term dynamical behavior of short-period comets. Icarus 108, 18-36 (1994).

55. Brož, M., Vokrouhlický, D., Morbidelli, A., Nesvorný, D. \& Bottke, W. F. Did the Hilda collisional family form during the late heavy bombardment? Mon. Not. R. Astron. Soc. 414, 2716-2727 (2011). 1109.1114.

56. Vokrouhlický, D. Diurnal Yarkovsky effect as a source of mobility of meter-sized asteroidal fragments. I. Linear theory. Astron. Astrophys. 335, 1093-1100 (1998).

57. Vokrouhlický, D. \& Farinella, P. The Yarkovsky Seasonal Effect on Asteroidal Fragments: A Nonlinearized Theory for Spherical Bodies. Astron. J. 118, 3049-3060 (1999). 
58. Čapek, D. \& Vokrouhlický, D. The YORP effect with finite thermal conductivity. Icarus 172, 526-536 (2004).

59. Farinella, P., Froeschlé, C. \& Gonczi, R. Meteorite Delivery and Transport. In Milani, A., di Martino, M. \& Cellino, A. (eds.) Asteroids, Comets, Meteors 1993, vol. 160 of IAU Symp., 205 (1994).

60. Alí-Lagoa, V. et al. Differences between the Pallas collisional family and similarly sized Btype asteroids. Astron. Astrophys. 591, A14 (2016).

61. Brož, M. \& Morbidelli, A. A study of 3-dimensional shapes of asteroid families with an application to Eos. Icarus 317, 434-441 (2019). 1810.04113.

62. Benz, W. \& Asphaug, E. Impact simulations with fracture. I - Method and tests. Icarus 107, 98 (1994).

63. Jutzi, M., Holsapple, K., Wünneman, K. \& Michel, P. Modeling asteroid collisions and impact processes. In Asteroids IV, 679-699 (University of Arizona Press, Tucson, 2015).

64. Ševeček, P. et al. SPH/N-Body simulations of small $(\mathrm{D}=10 \mathrm{~km})$ asteroidal breakups and improved parametric relations for Monte-Carlo collisional models. Icarus 296, 239-256 (2017).

65. Benz, W. \& Asphaug, E. Impact simulations with fracture. I - Method and tests. Icarus 107, 98 (1994).

66. Richardson, D. C., Quinn, T., Stadel, J. \& Lake, G. Direct Large-Scale N-Body Simulations of Planetesimal Dynamics. Icarus 143, 45-59 (2000). 
67. Tillotson, J. H. Metallic equations of state for hypervelocity impact. Gen. Atomic Rep. GA$3216(1962)$.

68. von Mises, R. Mechanik der festen krper im plastisch- deformablen zustand. Nachrichten von der Ges. der Wissenschaften zu Göttingen, Math.-Phys. Klasse 582-592 (1913).

69. Grady, D. \& Kipp, M. Continuum modelling of explosive fracture in oil shale. Int. J. Rock. Mech. Min. 17, 147-157 (1980).

70. Benz, W. \& Asphaug, E. Simulations of brittle solids using smooth particle hydrodynamics. Comput. Phys. Commun. 87, 253-265 (1995).

71. Bottke, W. F. \& Greenberg, R. Asteroidal collision probabilities. Geophys. Res. Lett. 20, 879-881 (1993).

72. Bottke, W. F. et al. Linking the collisional history of the main asteroid belt to its dynamical excitation and depletion. Icarus 179, 63-94 (2005).

73. Benz, W. \& Asphaug, E. Catastrophic Disruptions Revisited. Icarus 142, 5-20 (1999). arXiv: astro-ph/9907117.

74. Vernazza, P. et al. The impact crater at the origin of the Julia family detected with VLT/SPHERE? Astron. Astrophys. 618, A154 (2018).

75. Vilas, F. \& Gaffey, M. J. Phyllosilicate absorption features in main-belt and outer-belt asteroid reflectance spectra. Science 246, 790-792 (1989). 
76. Fornasier, S., Lantz, C., Barucci, M. A. \& Lazzarin, M. Aqueous alteration on main belt primitive asteroids: Results from visible spectroscopy. Icarus 233, 163-178 (2014).

77. Spectral reflectance properties of carbonaceous chondrites 4: Aqueously altered and thermally metamorphosed meteorites. Icarus 220, 586-617 (2012).

78. Lantz, C. et al. Ion irradiation of carbonaceous chondrites: A new view of space weathering on primitive asteroids. Icarus $\mathbf{2 8 5}$, 43-57 (2017).

79. Rivkin, A. S., Asphaug, E. \& Bottke, W. F. The Case of the Missing Ceres Family. Icarus $1-54(2014)$.

80. Castillo-Rogez, J. et al. Insights into Ceres's evolution from surface composition. Meteorit. Planet. Sci 53, 1820-1843 (2018).

81. Howard, K. T., Benedix, G. K., Bland, P. A. \& Cressey, G. Modal mineralogy of CM chondrites by X-ray diffraction (PSD-XRD): Part 2. Degree, nature and settings of aqueous alteration. Geochim. Cosmochim. A. 75, 2735-2751 (2011).

82. Alí-Lagoa, V. et al. Physical properties of B-type asteroids from WISE data. Astron. Astrophys. 554, A71 (2013). 1303.5487.

83. Castillo-Rogez, J. C. et al. Iapetus' geophysics: Rotation rate, shape, and equatorial ridge. Icarus 190, 179-202 (2007).

84. Schmidt, B. E. \& Castillo-Rogez, J. C. Water, heat, bombardment: The evolution and current state of (2) Pallas. Icarus 218, 478-488 (2012). 
85. Opeil, C. P., Consolmagno, G. J. \& Britt, D. T. The thermal conductivity of meteorites: New measurements and analysis. Icarus 208, 449-454 (2010).

86. Dufresne, E. R. \& Anders, E. On the chemical evolution of the carbonaceous chondrites. Geochim. Cosmochim. A. 26, 1085-1114 (1962).

87. Bland, P. A. \& Travis, B. J. Giant convecting mud balls of the early solar system. Sci. Advances 3, e1602514 (2017).

88. Carry, B. et al. Homogeneous internal structure of CM-like asteroid (41) Daphne. Astron. Astrophys. 623, A132 (2019). 1901.01890.

89. Alí-Lagoa, V. et al. Differences between the Pallas collisional family and similarly sized Btype asteroids. Astron. Astrophys. 591, A14 (2016).

90. Hanuš, J. et al. Shape, size, physical properties and nature of low-perihelion near-Earth asteroid (3200) Phaethon. In AAS/Division for Planet. Sci. Meeting Abstr., vol. 48 of AAS/Division for Planet. Sci. Meeting Abstr., 516.08 (2016).

91. Masiero, J. R., Wright, E. L. \& Mainzer, A. K. Thermophysical Modeling of NEOWISE Observations of DESTINY+ Targets Phaethon and 2005 UD. Astron. J. 158, 97 (2019). 1907. 04518.

92. Kareta, T. et al. Rotationally Resolved Spectroscopic Characterization of Near-Earth Object (3200) Phaethon. Astron. J. 156, 287 (2018). 1810. 11157. 
93. Ito, T. et al. Extremely strong polarization of an active asteroid (3200) phaethon. Nat. Commun. 9, 2486 (2018). URL https://doi .org/10.1038/s41467-018-04727-2.

94. Cellino, A. et al. On the calibration of the relation between geometric albedo and polarimetric properties for the asteroids. Mon. Not. R. Astron. Soc. 451, 3473-3488 (2015). 1506.00554.

95. Lupishko, D. F. Generalized Calibration of the Polarimetric Albedo Scale of Asteroids. Solar Syst. Res. 52, 98-114 (2018).

96. Devogèle, M. et al. The phase-polarization curve of asteroid (3200) Phaethon. Mon. Not. R. Astron. Soc. (2018). 1806.05277 .

97. Shinnaka, Y. et al. Inversion Angle of Phase-polarization Curve of Near-Earth Asteroid (3200) Phaethon. Astrophys. J. Lett. 864, L33 (2018). 1808.05746.

98. Takir, D. et al. 3-um Spectroscopy of Asteroid (3200) Phaethon: Implications for B-Asteroids. In Lunar and Planet. Sci. Conf., vol. 49 of Lunar and Planet. Sci. Conf., 2624 (2018).

99. Arai, T. et al. DESTINY+ Mission: Flyby of Geminids Parent Asteroid (3200) Phaethon and In-Situ Analyses of Dust Accreting on the Earth. In Lunar and Planet. Sci. Conf., vol. 49 of Lunar and Planet. Sci. Conf., 2570 (2018). 